\title{
A numerical-experimental method for damage location based on rotation fields spatial differentiation
}

\author{
H.M. Reis Lopes ${ }^{\text {a }}$, J.V. Araújo dos Santos ${ }^{\text {b,* }}$, C.M. Mota Soares ${ }^{b}$, R.J. Miranda Guedes ${ }^{c}$, M.A. Pires Vaz ${ }^{\text {c }}$ \\ ${ }^{a}$ ESTIG, Instituto Politécnico de Bragança, Campus de Sta. Apolónia, Apartado 134, 5301-857 Bragança, Portugal \\ b IDMEC/IST, Instituto Superior Técnico, Av. Rovisco Pais, 1049-001 Lisboa, Portugal

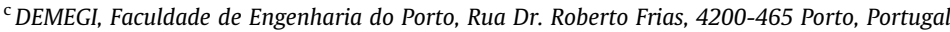

\section{A R T I C L E I N F O}

\section{Article history:}

Received 27 November 2008

Accepted 5 September 2010

Available online 16 April 2011

\section{Keywords:}

Multiple damage location

Laminated plate

Curvature field

Shear interferometry

Gaussian function derivative

Filtering techniques

\begin{abstract}
A B S T R A C T
This paper presents a structural damage location method that decreases the number of spatial differentiations needed to compute modal curvature fields. The method is numerically and experimentally applied to isotropic and laminated rectangular plates, respectively. A speckle shear interferometer is used to measure the rotation fields of the laminated plate, while the isotropic plate is analysed by finite elements. It was found that the Gaussian differentiation is the most suited technique to compute the curvature fields. It is also demonstrated the superior performance of the curvature method using measured rotation fields instead of measured displacement fields.
\end{abstract}

(c) 2010 Civil-Comp Ltd and Elsevier Ltd. All rights reserved.

\section{Introduction}

The interest in predictive structural monitoring, aiming at damage early detection, has been motivated by, amongst other factors, the risk of human lives loses, due to unpredictable structural failures, such as airplane crashes and bridges or buildings collapses. Damage detection and localisation aims at preventing the structures failure by repairing or replacing the damaged component. The need of global methods for damage detection has been essentially motivated by aeronautical and aerospace applications. In these applications, the less critical components have been progressively replaced by components manufactured with composite materials. Although their high specific stiffness and strength, compared to those of metals, composite materials are more sensitive to certain type of damages and present different kinds of defects or damage mechanisms. The main damage types in laminated composites are interlaminar debonding, micro-cracks and micro-buckling, besides inclusions. These internal damages usually result from manufacturing processes or in service loadings. Delamination or interlaminar debonding is undetected by visual means and, therefore, one of the most critical type of damage, being also the most common in aeronautical components.

There are several types of methods which use dynamic characteristics, namely those based on modal frequencies, displacements,

\footnotetext{
* Corresponding author. Tel.: +44958 8846.

E-mail address: viriato@ist.utl.pt (J.V. Araújo dos Santos).
}

rotations and curvatures [1-14]. Among these, the global methods based on curvature changes can be given some prominence. Pandey et al. [3] introduced the mode shapes curvature method. In order to localise damage in beams, the proposed method uses the difference in the curvature fields associated with mode shapes in the undamaged and damaged states. The curvatures are extracted by applying second order central finite differences to the mode shapes. Contrary to the mode shapes, one can prove that the higher changes in mode shapes curvatures are coincident with the damage region and their magnitudes are proportional to its severity. An extension of the curvature differences method was proposed by Sampaio et al. [7], who also used second order central finite differences to compute the frequency response functions curvatures. Recently, Guan and Karbhari [12] stated that the computation of the curvatures by numerical differentiation is the main cause for the poor performance of the curvature method, in particular under sparse and noisy measurements. In order to improve the numerical differentiation results they proposed the use of a fourth order central difference method, instead of applying the second order central difference. The damage index method formulation presented by Stubbs et al. [4] is also based on mode shapes curvatures information. This damage index relates the undamaged and damaged curvature fields in each segment of the structure. Its deviation from the indices normal distribution average is used as an indicator for the identification of the probable damaged region. Also in the context of curvature methods, Ratcliffe [5] developed a procedure that does not require undamaged structure information. The curvature 